\title{
Mohamed Ridha Bouguerra et Sabiha Bouguerra, Histoire de la littérature du Maghreb, Littérature francophone
}

Paris, Ellipses, coll. « Littératures du monde », 2010, 255 p.

\author{
Alaeddine Ben Abdallah \\ Université d'Ottawa
}

Les monographies et les anthologies consacrées à la littérature maghrébine sont peu nombreuses. À l'instar de Jean Déjeux, de Jacqueline Arnaud, de Charles Bonn, d'Albert Memmi, d'Abdelkébir Khatibi et de quelques autres, les auteurs de ce livre, Mohamed Ridha Bouguerra et Sabiha Bouguerra, examinent, comme par radioscopie, le corps littéraire de la francophonie maghrébine. D'emblée, dès l'introduction, la dimension historique et, plus précisément, le contexte socio- 
politique duquel cette littérature a émergé est abordé, à l'image de la majorité des études qui y sont consacrées, sous l'angle de la langue, en prenant soin de distinguer la littérature ethnographique, dépourvue de critique, du début du XX ${ }^{\text {e }}$ siècle, et la littérature contestataire, qui a pris naissance dans les années 1950. Aussi les auteurs précisent-ils leur intention d'évacuer cette littérature dite folklorique de leur ouvrage et de ne se pencher que sur le corpus pré- et post-indépendances ou ce que les auteurs nomment "littérature de revendication ». Pour ce faire, ils suivent un plan à la fois chronologique et thématique qui leur permet de couvrir les aspects les plus saillants du champ littéraire maghrébin francophone, si champ il ya.

Dans la première partie du livre, « La période coloniale. L'émancipation d'une littérature critique», est exposée la dimension dissidente par laquelle nombre d'écrivains maghrébins tentent de rompre avec l'ancienne génération et ses codes sociopolitiques. Les figures de proue de cette contestation de l'ordre établi sont bien évidemment Driss Chraïbi et Rachid Boudjedra, qui « déclarent même la mort du père » (p. 24), mais aussi Mouloud Mammeri, Mouloud Feraoun, Mohamed Dib, Malek Hddad et Kateb Yacine. Il faut voir par là, au-delà de l'image du père, une contestation de la société patriarcale, mais encore, et ce point est quelque peu ignoré dans l'ouvrage, de l'autorité divine, laquelle accapare les attributs du père.

Parallèlement à cette volonté de se révolter contre «la férule paternelle » (p. 25) dans tous les sens du terme, naît une envie pressante de rencontrer l'Autre culturel, et ce, malgré le contexte colonial qui régissait cette relation. De cette rencontre 
découlent une déception et un désenchantement existentiel et l'écrivain et intellectuel se trouvent en position du solitaire, tiraillé qu'il est entre la modernité occidentale et les codes archaïques de sa société d'origine, ce qui transparaît dans l'hexis et l'ethos de la plupart des personnages dont, entre autres, le fameux protagoniste Driss dans Le Passé simple de Driss Chraïbi ou encore Mokrane dans La Colline oubliée de Mouloud Mammeri. Cependant, il est mentionné que la critique qu'entreprirent certains écrivains maghrébins de leurs propres traditions et «la dénonciation des valeurs périmées d'une société archaïque et figée dans des traditions séculaires» (p. 29) étaient une condition pour se soulever et faire face au colonisateur, sans quoi il n'y aurait pas eu de révolution. Cet engagement politique et idéologique, loin de tout manichéisme, a valu à ces écrivains le rôle d'«écrivain public », à partir duquel Kateb Yacine et, plus tard, Tahar Ben Jelloun se positionnaient au sein du champ littéraire qui émergeait. En effet, sous-jacente aux préoccupations poétiques, la dimension réaliste et documentaire chez des écrivains tels que Mohamed Dib montre le dénuement et l'injustice sociale que subit le peuple. C'est dans ce contexte tendu que les événements de la guerre d'Algérie furent dépeints avec toute la verve et la ténacité d'un Malek Haddad et de tant d'autres qui instituèrent de véritables « œuvres de combat » (p. 43), à l'instar de Kateb Yacine - dont le personnage éponyme Nedjma est une métaphore du pays convoité par tous - qui « a fait entrer dans la modernité la littérature maghrébine francophone » (p. 46).

À cette première période succède celle de la deuxième génération, la «littérature de la désillusion » (p. 51). En effet, les espoirs des indépendances ont vite laissé place à un sentiment de désenchantement politique. La décolonisation et 
ses multiples échecs marquent la période des années 1970, où des écrivains tels que Nabil Farès, Rachid Boudjedra, Abdelkébir Khatibi, Tahar Ben Jelloun, Abdelwahab Meddeb, etc., réservaient leurs virulentes critiques aux pouvoirs en place, mais également aux "sociétés sclérosées" (p. 52). Toutefois, cette désillusion se démarque par une certaine prise de distance de la dimension réaliste et militante de la première génération, laquelle fut remplacée par une portée poétique, voire onirique, sans toutefois désamorcer la colère et l'invective de ces jeunes écrivains. Cet engagement d'une autre nature s'exprime «à travers le refus des codes littéraires établis, la subversion des genres traditionnels et la revendication d'une écriture anticonformiste, provocatrice même » (p. 53). Dans ce contexte, voit le jour un collectif d'écrivains, de poètes et de penseurs marocains réunis autour de la revue Souffle, qu'Abdellatif Laâbi a fondée et qui, au-delà des préoccupations purement poétiques, remettait souvent en cause les institutions établies et s'inscrivait dans un « rejet radical de tout conformisme» (p.57). La revue marque l'avènement d'une nouvelle orientation littéraire allant de la dimension baroque, voire iconoclaste, de l'œuvre de Khaïr-Eddine, jusqu'à l'écriture fantasmatique et asyndétique de Meddeb en passant par l'œuvre "elliptique» (p.64) et conceptuelle de Khatibi. Pour cette génération, l'essentiel est d'exprimer, à travers une langue altérée et un style réinventé, « le malaise d'un intellectuel arabe qui n'accepte pas la confiscation de l'indépendance et l'instauration » (p. 68) de régimes autoritaires.

Divisant la partie de «l'ère des indépendances » en deux, les auteurs de l'ouvrage rangent dans la deuxième section les personnages marginaux que les écrivains érigent en véritables anti-héros. Cette littérature se cantonne dans des asiles 
psychiatriques et la folie devient son principal logos. De L'Escargot entêté à Moha le fou Moha le sage et à Harrouda, mais aussi avec toute l'œuvre de Ben Jelloun, en passant par L'Insolation de Rachid Boudjedra, les romanciers mettent en avant des personnages qui, si fous soient-ils, montrent clairement les travers de la société et disent l'indicible. Ils se servent ainsi de la figure de la déraison pour « revisiter le passé et juger le présent par un monstre comme Tombéza, se donn[a]nt le droit à une parole sans entrave » (p. 74). Ce détour par la folie et par les figures marginales de la société permettrait aux écrivains de mieux faire passer leur indignation vis-à-vis de la décolonisation et des régimes politiques dans les années 1970. C'est ainsi que les écrivains de cette génération désenchantée se reconnaissent entre eux et, à travers leurs personnages, ils font référence à leurs confrères, tels que le Tunisien Anouar Attia, qui évoque dans De $A$ jusqu'à $T$ le personnage Moha en référence à Moha le fou moha le sage de Ben Jelloun afin de rendre hommage à ce dernier, mais surtout dans le but de souligner "l'impasse à laquelle se trouvent acculés tous ceux de sa génération passés par les universités françaises, déchirés entre tradition et modernité et s'adaptant mal dans une société répressive » (p. 107).

Cependant, dans les années 1990, qualifiées d'années de plomb, la veine provocatrice, dans la littérature maghrébine, loin de s'estomper, prend au contraire une autre tournure. Dans un contexte socio-politique marqué par l'intégrisme religieux et la guerre civile en Algérie, les écrits littéraires y trouvent leur cible et leurs auteurs ne s'épargnent pas les dangers qui y sont liés en fustigeant la haine propagée par l'intégrisme, mais aussi par le despotisme de l'État. Ainsi, fictions et essais deviennent un vecteur essentiel de cette révolte contre tout système 
autocratique, à l'instar de FIS de la haine de Rachid Boudjedra, De La barbarie en général et de l'intégrisme en particulier de Rachid Mimouni ou encore L'Invention du désert et Les Vigiles de Tahar Djaout, assassiné pour ses idées par les extrémistes. Assia Djebar, dans Oran, langue morte, ressasse les crimes des islamistes.

Sur un ton aussi accusateur que les écrivains précédemment cités, mais plus versés dans le réalisme, figurent le cinéaste et écrivain Merzak Allouache et Yasmina Khadra, qui met en scène le terrorisme algérien dans Morituri et dans L'Automne des chimères, mais aussi Boualem Sansal qui n'hésite pas, dans Le Serment des barbares, à relier le terrorisme des années 1990 en Algérie aux origines de la lutte pour l'indépendance, mettant par là au rang des accusés aussi bien les «fous de Dieu » que le FLN. Pour cette raison, cet écrivain «iconoclaste » incarne "l'ennemi juré des fanatiques en train de gangréner la société algérienne, mais aussi le critique indigné, ironique et amer de l'arbitraire érigé en règle par les services de l'administration algérienne » (p. 122), ce qui lui vaut d'être licencié de son poste de haut-fonctionnaire de l'État.

Dans la même lignée que Sensal et Khadra, de nouveaux écrivains viennent à la littérature, mus par cette « descente aux enfers» (p.124) dans laquelle sombre la société algérienne. Aussi iconoclastes que les deux écrivains cités ci-dessus, Anouar Benmalek, Aïssa Khelladi et Mourad Djebel marquent la génération actuelle d'écrivains et accentuent encore plus la révolte contre le système intégriste et le système étatique dans un réalisme cru, décrivant l'horreur de la guerre des années 1990. Tel est le cas Des Amants désunis de Anouar Benmalek et de Rose d'abîme de Aïssa Khelladi, qui inscrit sa pièce de 
théâtre, dont la structure éclatée rappelle Nedjma de Kateb Yacine, dans un va-et-vient entre réalisme et symbolisme avec, comme toile de fond, la guerre civile algérienne.

Parallèlement à cette littérature du «témoignage » propre à l'Algérie des années 1990-2000, une production dont le motif principal est l'exil se développe et regroupe des écrivains maghrébins de la diaspora. Dans cette voie, Fouad Laroui met en avant l'exil comme quête d'une liberté individuelle. Après cet exil surgit la réalité des émigrés en Europe qui font face au racisme et à l'exclusion sociale. Les œuvres classiques maghrébines en font un écho retentissant, telles que Les Boucs de Chraïbi et Le Polygone étoilé de Yacine. Malek Haddad décrit cette masse d'émigrés, dans La Dernière impression, comme des êtres solitaires et presque invisibles pour les autres (p. 139). Tahar Ben Jelloun a fait de la condition de l'émigré un objet d'étude psychosociologique dans son essai La Plus Haute des solitudes et plus tard dans La Réclusion solitaire, où il «multiplie les images de ceux qui s'étaient confiés à lui » (p. 143). Il va sans dire que bon nombre d'émigrés maghrébins sont confrontés au désarroi ainsi qu'à la misère matérielle et affective, à l'instar des trois vieillards algériens dans le roman d'Abdekader Djemaï, Gare du nord. Mais le roman de Rachid Boudjedra, Topographie idéale pour une agression caractérisée, est probablement celui qui dépeint avec « le plus de réalisme et de véracité la situation dramatique des ouvriers maghrébins émigrés en France » (p. 141).

Parmi ces écrivains de la diaspora figurent ceux qu'on appelle «beurs» et qui forment la seconde génération d'émigrés maghrébins. Le discours de leurs productions littéraires diffère quelque peu de celui des précédents et inscrit 
le conflit culturel entre les parents émigrés et leurs enfants nés en France. Azouz Begag, Mehdi Charef, Faïza Guène et Akli Tadjer évoquent la France multiethnique et revendiquent leur métissage culturel malgré leurs enfances difficiles. Toutefois, cet ancrage sociopolitique ne les empêche pas de poser un regard sur les clandestins et les sans-papiers, comme c'est le cas dans le roman de Akli Tadjer Bel-Avenir.

Si des écrivains mettent en avant le conflit générationnel et expriment ainsi leur révolte mais aussi leur désenchantement, d'autres fuient le présent morose en chantant la gloire d'un passé révolu. Tel est le cas de la plupart des écrivains tunisiens francophones, de Chems Nadir qui convoque, à travers sa poésie, « les siècles d'or de la civilisation arabo-musulmane afin de mettre en exergue les difficultés que rencontrent, aujourd'hui, les héritiers de cette brillante civilisation » (p.156), à Fawzi Mellah qui remonte jusqu'aux origines de Carthage (Le Conclave des pleureuses), en passant par Hédi Bouraoui qui évoque la Tunisie romaine (Retour à Thyna) et Alia Mabrouk qui revisite l'histoire romaine du point de vue des vaincus (Blé de Dougga).

La dernière partie de l'ouvrage est consacrée aux « voix féminines du Maghreb ou la libération par l'écriture ». Faut-il rappeler que les voix féminines se taillent la part du lion au cœur du champ littéraire maghrébin francophone et qu'elles s'exportent beaucoup mieux que les voix masculines? Le discours féministe qui jalonne leurs écrits en est le blason et se donne pour mission de dénoncer les pratiques machistes et misogynes des sociétés maghrébines. Force est de relever que cette littérature féminine a vu le jour bien avant les années 1950 et a donc devancé sa consœur masculine bien qu'elle fût 
l'apanage de quelques femmes pieds-noirs, métisses ou converties, telles qu'Élisa Chimenti, Elissa Rhaïs et Marie-Louise Taos Amrouche - considérée comme la première écrivaine kabyle -, dont les écrits remontent aux années 1930. Cette « littérature de combat» (p. 187), initiée par Taos Amrouche, a produit au sein de ce champ des agentes au discours véhément qui militent «pour l'émancipation et l'égalité de la femme» (p. 187). En Tunisie, Souâd Guellouz et Jalila Hafsia ont écrit les premières autobiographies romancées (respectivement Les Jardins du nord et Instants de vie); Hélé Béji a interrogé la première «les notions ou concepts d'identité culturelle, nationalisme et modernité » (p. 190) dans Désenchantement national; Azza Filali, Amina Saïd et Faouzia Zouari ont montré une certaine sensibilité existentielle, doublée du fameux tiraillement entre le pays d'origine et le pays d'accueil, tel qu'il est exposé dans La Retournée de Fawzia Zouari.

$\mathrm{Au}$ Maroc, les écrivaines mènent un combat pour la dignité et condamnent l'oppression ancestrale dont les femmes sont l'objet. Fatima Mernissi, essayiste et sociologue, s'est imposée comme la spécialiste de la sexualité dans les sociétés musulmanes. Siham Benchekroun dénonce l'hypocrisie sociale dans les relations intersexuelles (Oser vivre). Nadia Chafik replonge dans l'histoire coloniale, mais se préoccupe également de l'affrontement entre tradition et modernité dans le Maroc d'aujourd'hui.

Cependant, vu le contexte socio-historique particulier, c'est l'Algérie qui s'impose loin devant, avec des écrivaines telles qu'Assia Djebar, qui siège à l'Académie française et qui a donné la parole aux voix féminines récluses, ou encore Yamina Mechakra et Anna Gréki, qui ont dépeint, dans une prose 
poétique et des poèmes, les affres de la guerre de libération, Malika Mokeddem, qui mêle autobiographie et fiction, ainsi que Maïssa Bey, Leïla Sebbar et Bouthaïna Azami-Tawil, qui s'attaquent à la misogynie de la société algérienne ainsi qu'à la violence du terrorisme et s'évertuent à condamner le « retour de la barbarie et de l'instrumentalisation de la religion aux fins déclarées d'affermir encore davantage la mise au pas de la moitié féminine de la société » (p. 210).

L'ouvrage fait le tour, d'une manière concise et intelligente, des grands axes historiques et thématiques que parcourt la littérature francophone du Maghreb. Loin des anthologies classiques, cet ouvrage a le mérite de raconter la littérature maghrébine de façon attrayante. Les seuls griefs que nous faisons aux auteurs de cet ouvrage tiennent à ce qu'à force de vouloir jongler entre le découpage historique des périodes littéraires et la partition thématique, les époques s'entremêlent quelque peu, faisant en sorte que la référence aux mêmes écrivains revient à plusieurs reprises. En outre, le traitement réservé aux écrivaines ne se démarque guère des études sexistes classiques, qui cantonnent les femmes à une section à part. Il aurait fallu plutôt discuter de la dimension féministe, laquelle déborde le fait de genres au profit d'un discours particulier. Et dans cette catégorie, aussi bien les écrivains que les écrivaines pourraient être rangés en fonction de la veine féministe qui traverse leurs écrits. 